\title{
Doppelgespann investiert in Studienprogramm
}

D ie beiden Unternehmen Novartis Pharma und Leti Pharma haben die Verlängerung ihrer bereits seit 2005 bestehenden Zusammenarbeit bis zum Jahr 2022 beschlossen. Grund hierfür sind die positiven Ergebnisse der bisherigen Kooperation zwischen dem breit aufgestellten internationalen Konzern einerseits und dem auf den Allergiebereich spezialisierten spanischen Mittelständler andererseits. „Wir sehen, dass unser AllergologiePortfolio sehr gut von Ärzten und Patienten angenommen wird und unsere Produkte eine hohe Akzeptanz genießen“, erklärte dazu Dr. Dirk Kosche, Vorsitzender der Geschäftsführung von Novartis Pharma. „Damit ist es nur selbstverständlich“, so Kosche weiter, „den Vertrag zu verlängern, um Patienten im Allergiebereich langfristig erstklassige Produkte und ein breites Service-Angebot anzubieten."

Gleichzeitig haben die beiden Unternehmen ein umfangreiches Programm an Zulassungsstudien aufgesetzt. Damit kommen sie den Anforderungen der Therapieallergene-Verordnung, die eine Ausdehnung der Vorschriften zur Arzneimittelzulassung auf Allergenextrakte vorsieht, in vollem Umfang nach. „Wir begrüßen diesen Vorstoß des Gesetzgebers, einheitliche Standards für Therapieallergene zu fordern“, unterstrich Kosche. „Daher werden wir unsere Forschungsanstrengungen im Bereich Allergologie noch weiter intensivieren und in den nächsten Jahren gemeinsam mit Novartis Pharma mehr als 100 Millionen Euro in klinische Studien investieren “, ergänzte Dr. Jaime Grego, Geschäftsführer von Leti Pharma.

Konkret geplant sind über 20 plazebokontrollierte Doppelblindstudien mit den Produkten Depigoid ${ }^{\circledR}$ für die perenniale subkutane Immuntherapie, mit Depiquick $^{\circledR}$ für die subkutane Kurzzeitimmuntherapie und mit TOL SL plus für die sublinguale Immuntherapie. Alle Studien folgen hohen wissenschaftlichen Standards und werden gemäß den Anforderungen des Therapieallergene-
Zulassungsverfahrens des Paul-EhrlichInstitut durchgeführt. „Wir gehen davon aus, dass wir bis zur Entscheidung über die Zulassung für alle Formulierungen erfolgreich abgeschlossene Studien vorlegen können“, prognostizierte Grego. Bis dahin können alle Therapieallergene von Novartis zur spezifischen Immuntherapie weiter uneingeschränkt verwendet werden.

Bereits seit Jahren zeichnen sich Novartis Pharma und Leti Pharma durch ein besonderes Engagement in der Forschung aus. Die hohe Wirksamkeit und gute Verträglichkeit von Depigoid $^{\circledR}$ und Depiquick ${ }^{\circledR}$ ist beispielsweise bisher in 22 klinischen Studien mit 4.139 Patienten dokumentiert worden. Zudem bietet Novartis mit Depigoid $^{\circledR}$ Milbe ein Milbenallergoid mit einer Zulassung nach den neuen Zulassungsanforderungen und entsprechend den strengen WAO-Kriterien an.

Novartis Pharma ist Meister in der klinischen Forschung: 2009 belegte das Unternehmen mit 115 Studien zuzüglich 37 Phase-II- und -IIIb-Studien mit deutlichem Abstand Platz eins bei der Anzahl der klinischen Studien in Deutschland. Über 16.000 Patienten waren an den Studien beteiligt, davon rund 2.000 aus dem Indikationsbereich Dermatologie/Pneumologie. Auf dem Gelände von Leti Pharma nahe Madrid wird außerdem ständig an Innovationen gearbeitet. „Depigoid ${ }^{\circledR}$ und Depiquick ${ }^{\circledR}$ werden mit einem einzigartigen Aufreinigungsverfahren - der so genannten Depigmentierung - hergestellt. Damit werden allergologisch irrelevante Verunreinigungen besonders sorgfältig entfernt, was bei guter Verträglichkeit die Verabreichung hoher Allergendosen in kurzer Zeit ermöglicht", erläuterte Grego. Darüber hinaus betreibt das Unternehmen in seiner Einrichtung in Spanien auch das weltweit größte Milbenzuchtlabor.

red

Nach Informationen von Novartis

Pharma, Nürnberg

\section{Neues Antihistaminikum}

Für die Behandlung von allergischer Rhinitis und Urtikaria steht mit Urtimed ${ }^{\circledR}$ ein neues Antiallergikum des Unternehmens Dr. R. Pfleger zur Verfügung. Als Wirkstoff enthält es Rupatadin, das den Beschwerden auf zweifache Weise entgegentritt: Es weist nicht nur eine sehr hohe Affinität zum H1-Rezeptor auf, es wirkt zusätzlich antagonistisch auf den plättchenaktivierenden Faktor. Urtimed ${ }^{\circledR}$ eignet sich für Erwachsene und Jugendliche ab zwölf Jahren und ist rezeptpflichtig. Es in den Packungsgrößen 20, 50 und 100 Tabletten erhältlich. Der Preis liegt unter dem anderer führender rezeptpflichtiger Antihistaminika.

Nach Informationen von Dr. R. Pfleger, Bamberg

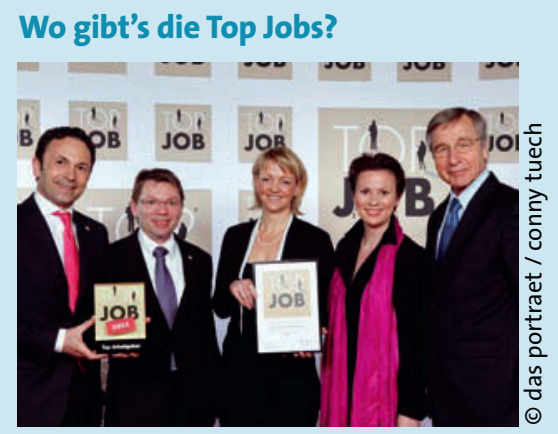

Mit dem Gütesiegel „Top Job“ wurden Ende Januar in Duisburg mittelständische Unternehmen für gutes Personalmanagement ausgezeichnet. Unter den besten Arbeitgebern Deutschlands erzielte Basilea Pharmaceutica den zweiten Platz in der Kategorie bis 100 Mitarbeiter. Das erst drei Jahre junge Unternehmen der forschenden Pharmabranche stellt Toctino $^{\circledR}$ her, ein systemisches AlitretinoinPräparat gegen chronisches Handekzem. Der ehemalige Arbeitsminister Wolfgang Clement, Mentor des Projekts, übergab den Preis. Er sagte: „Die Gewinner haben erkannt, wie wichtig gute Personalarbeit und eine engagierte Kommunikationspolitik sind. " Die Personalarbeit der Unternehmen wird jedes Jahr durch den Benchmarkingspezialisten Compamedia und die Universität St. Gallen ermittelt. Diesmal wurden auch die 36 Mitarbeiter von Basilea befragt. Sie lobten, dass sie eigene kreative Vorstellungen umsetzen könnten, und fühlten sich angekommen. „Dieses positive Meinungsbild macht uns stolz“, bewertete der Geschäftsführer Dr. Thomas Trilling den Erfolg.

Nach Informationen von Basilea Pharmaceutica, München 